(C)2016 IEEE. Reprinted, with permission, from V.M. Bhoopathy, M. Ben Haj Frej, S.R.E. Amalorpavaraj, and I. Shaik, "Localization and Mobility of Underwater Acoustic Sensor Nodes.” In Proceedings of 2016 Annual Connecticut Conference on Industrial Electronics, Technology \& Automation (CT-IETA), Bridgeport, CT, 2016.

This material is posted here with permission of the IEEE. Such permission of the IEEE does not in any way imply IEEE endorsement of any of the University of Bridgeport's products or services. Internal or personal use of this material is permitted. However, permission to reprint/republish this material for advertising or promotional purposes or for creating new collective works for resale or redistribution must be obtained from the IEEE by writing to pubs-permissions@ieee.org. By choosing to view this document, you agree to all provisions of the copyright laws protecting it. 


\section{Localization and Mobility of Underwater Acoustic Sensor Nodes}

Vignesh Mandalapa Bhoopathy, Mohamed Ben Haj Frej, Steve Richard Ebenezer Amalorpavaraj, Imran shaik

Department of Electrical Engineering

University of Bridgeport, Bridgeport, CT

\section{Abstract}

The Underwater Acoustic Sensor Network (UASN) is a network which consists of underwater wireless sensor nodes distributed randomly over the certain area of observation and communicate with each other using acoustic signals. UASN can be used in a wide variety of underwater applications, ranging from marine biology surveillance to earthquake and tsunami alert applications. Despite the fact that sensor networks are meant to collect data, in some applications, collection of data without a location of the occurrence becomes incoherent. Hence, localization for UASN has become an enthralling research topic in the recent years. However, there are several obstacles coming from the acoustic channel like propagation delay, limited energy sources, high bit error rate, and limited underwater harvesting techniques making the localization of UASN quite challenging. Therefore, in this poster we are proposing a distributed localization scheme which employs two-way time of arrival estimation method to calculate distance and avoid the requirement of time synchronization.

\section{Introduction}

Research on the Underwater Acoustic Sensors has seen rapid growth in recent years because of their wide prospects of potential applications ranging from exploration of rare minerals situated in the deep oceans, environmental monitoring, disaster warning systems, target monitoring systems, underwater habitat monitoring and oceanographic data gathering. Underwater wireless sensor networks behave as bridge between the virtual information technology and real world phenomena. Optimal performance of the networks requires a balance number of working nodes, energy efficiency of the network and lifetime of the network, so the number of functional nodes in the network are very crucial for the operation of the network as a whole.

\section{Problem Identification}

The Distributed localization scheme employs two-way time of arrival based estimation method to avoid the requirement of time synchronization. Only the ordinary nodes are localized since the beacon or anchor nodes are already localized. This method considers only the situations in which the nodes are idle, but node mobility is not considered during time synchronization.

The problem arises with the movement of the node during localization period. Thus, a suitable underwater event-driven localization scheme based on mobility prediction has to be established to overcome the effects of mobility of nodes.

\section{Methodology}

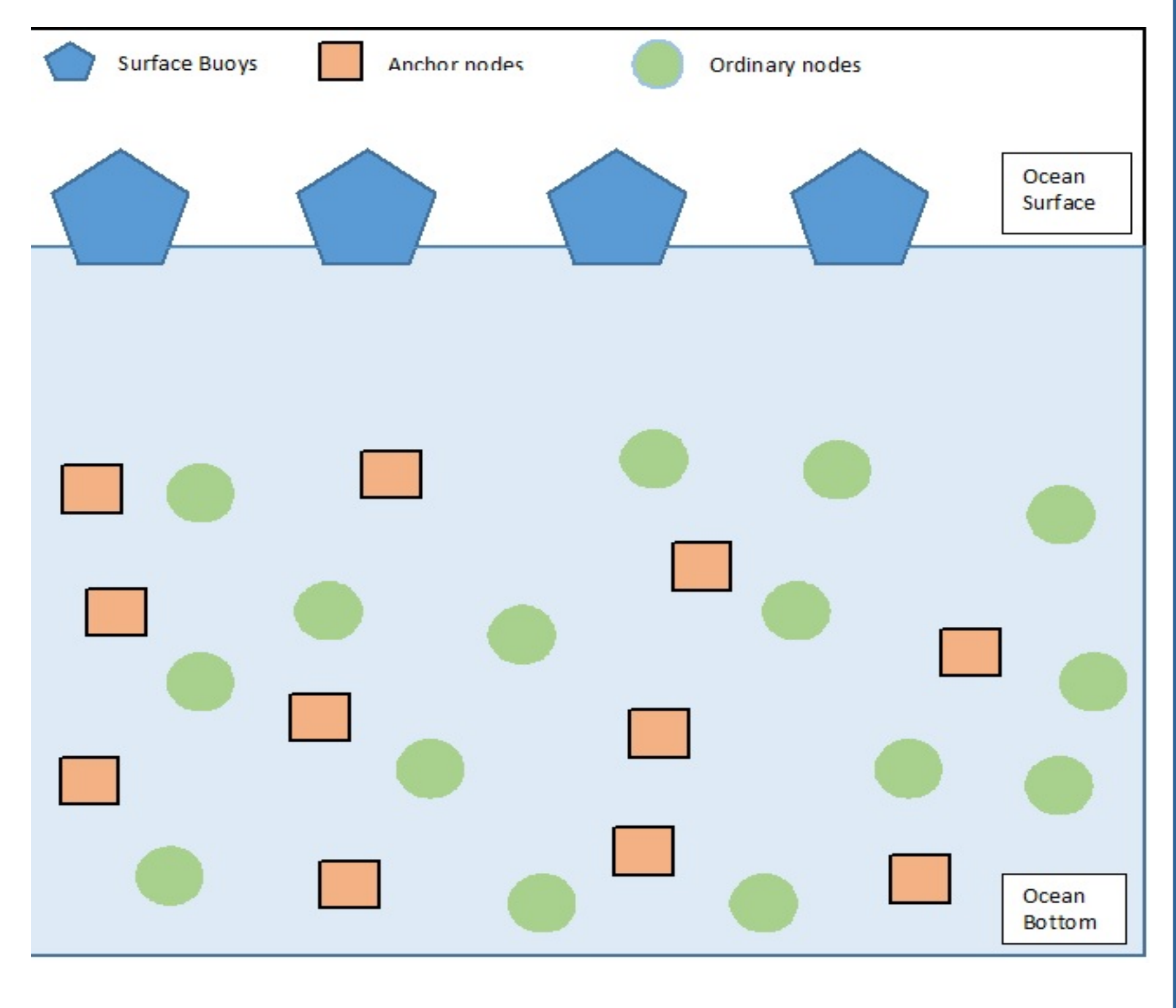

- In this approach, we consider an underwater scenario in which we randomly place the ordinary nodes, the surface buoys and anchor nodes.

- The surface buoys are fixed with a GPS receiver to get the exact location with the help of a satellite.

- The surface buoys send their location data to the anchor nodes, which are also known as beacon nodes. They receive their location information with the help of surface buoys and localize themselves with the other anchor nodes.

Ordinary nodes are the sensing nodes which are also present in the underwater.

All these ordinary nodes are non-localized. In order for the ordinary nodes to get localized, they must obtain location information from the anchor nodes, which is when they become reference nodes for the other non-localized nodes.

\section{Proposed Solution}

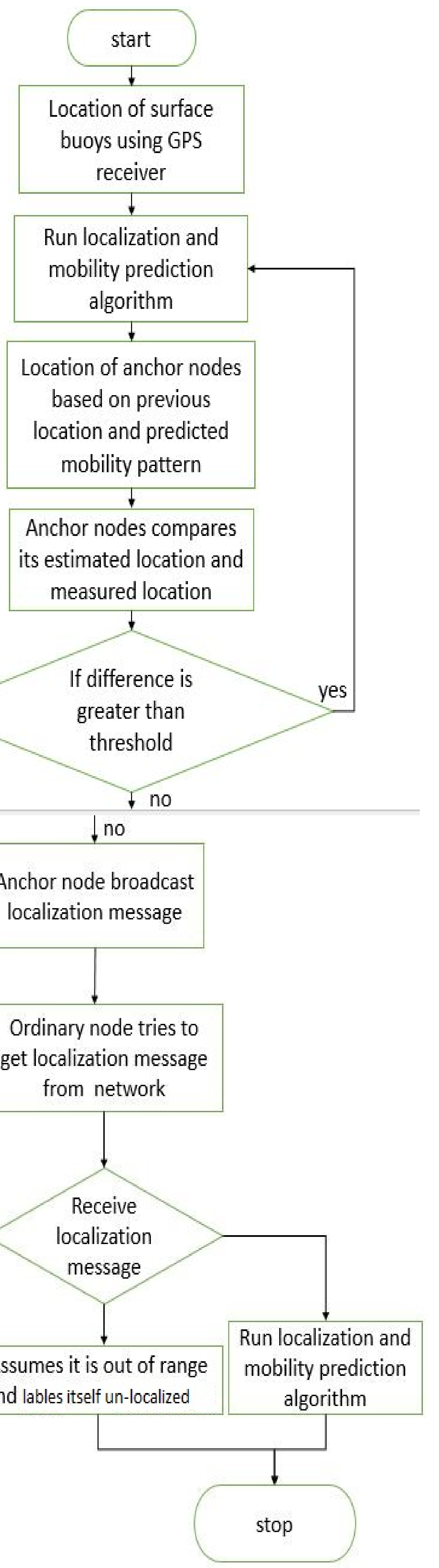

- Here a mobility based localization scheme is explained.

- There are three types of nodes in this underwater wireless sensor network, namely anchor nodes, surface buoys and ordinary nodes which are spread randomly in water.

- Surface buoys receive their location information from GPS receiver.
- The information about the location of anchor nodes will be calculated with respect to the location information of surface buoys and information about the position of ordinary nodes is calculated in accordance to the location of anchor nodes.

- Underwater sensors are subject to continuous movement because of water currents and dispersion.

- Objects near seashore represents periodic pattern because of these tides and the underwater objects tend to have a group movement property as the motion of a single object is correlated to the motion of other objects.

- The mobility in underwater wireless sensors is emphasized by adopting a kinematic mobility model.

- The proposed method is divided into two portions, in the first part anchor nodes localize and in the second part ordinary nodes are localized.

- In this network, surface buoys act as satellites and localize anchor nodes.

- The anchor nodes are superior to ordinary nodes because of their processing and computational properties.

- Therefore, the mobility tracking algorithm is to be implemented on them, but the ordinary nodes does not have such properties therefore recursive range based scheme can be used.

\section{Conclusion}

In this poster, we have considered the mobility prediction algorithm for the localization of ordinary nodes and anchor nodes in underwater sensor network. The localization of ordinary nodes is done by estimating the path of the ordinary node and previous location. The location is estimated with the help of localization message from the anchor nodes. This approach overcomes the mobility problem, thus reducing the communication cost and the overall delay in the network

\section{References}

Kundu, S. and P. Sadhukhan. Design and implementation of a time synchronization-free distributed localization scheme for underwater acoustic sensor network in Applications and Innovations in Mobile Computing (AIMoC), 2015. 2015. IEEE.

on scheme based on mobility prediction for underwater wireless sensor networks. in Control and Decision Conference (2014 CCDC), The 26th Chinese. 2014. IEEE.

Ko, J.H., J. Shin, and C. Kim. Localization of Sensor Nodes in Underwater Acoustic Sensor Networks Using Two Reference Points. in ICOIN. 2008.A

Zhou, Z., et al., Scalable localization with mobility prediction for 2011. 10(3): p. 335-348.

and Localization Design For

Douik, A., et al., Robust Node Estimation and Topology Discovery Algorithm in Large-Scale Wireless Sensor Networks. arXiv preprint arXiv:1508.04921, 2015 .

. International Conference on 2013 JEEE.

. Engineering and Information \& Communication Technical (ICEEICT), 2014 International Conference on. 2014. IEEE. Cheng, X., et al., Silent positioning in underwater acoustic sensor networks. IE 1756-1766.

10.Zhu, Z., et al. A multi-hop localization algorithm in underwater wireless sensor networks. in Wireless Communications and Signal Processing (WCSP), 2014 Sixth International Conference on. 2014. IEEE.

1.Yu, C.H., et al. Sensor localization algorthms in Underwater wireless sensor networks. in ICCAS-SICE, 2009. 2009. IEEE.

.Zhou, Z., J.-H. Cui, and S. Zhou, Efficient localization for large-scale underwater sensor networks. Ad Hoc Networks, 2010. 8(3): p. 267-279. Meiqin, L., G. Xiaodong, and Z. Senlin. Localization based on best spatial correlation distance mobility prediction for underwater wireless 2015. IEEE.

.Bhuvaneswari, P., et al. An efficient mobility based localization in underwater sensor networks. in Computational Intelligence and Conference on. 2012. IEE 